Accepted Manuscript

\title{
BIRTHS FROM EMBRYOS WITH HIGHLY ELEVATED LEVELS OF MITOCHONDRIAL DNA
}

Andrea R. Victor, Darren K. Griffin, David K. Gardner , Alan J. Brake, Christo G. Zouves, Frank L. Barnes , Manuel Viotti

PII:

DOI:

Reference:

To appear in:

Received date: Revised date:

Accepted date:
S1472-6483(19)30373-6

https://doi.org/10.1016/j.rbmo.2019.03.214

RBMO 2168

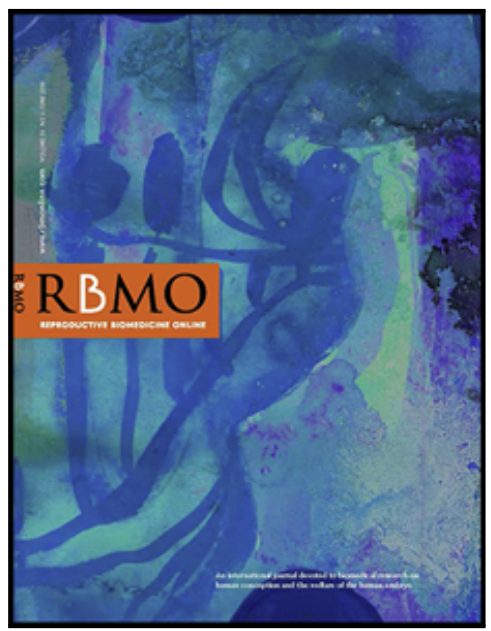

Please cite this article as: Andrea R. Victor, Darren K. Griffin, David K. Gardner, Alan J. Brake , Christo G. Zouves, Frank L. Barnes, Manuel Viotti, BIRTHS FROM EMBRYOS WITH HIGHLY ELEVATED LEVELS OF MITOCHONDRIAL DNA, Reproductive BioMedicine Online (2019), doi: https://doi.org/10.1016/j.rbmo.2019.03.214

This is a PDF file of an unedited manuscript that has been accepted for publication. As a service to our customers we are providing this early version of the manuscript. The manuscript will undergo editing, typesetting, and review of the resulting proof before it is published in its final form. Please note that during this process changes will be made and errors may be discovered which could affect the content. All legal disclaimers that apply to the journal pertain. 


\section{BIRTHS FROM EMBRYOS WITH HIGHLY ELEVATED LEVELS OF MITOCHONDRIAL DNA}

Andrea R. Victor ${ }^{\text {a,b }}$, Darren K. Griffin ${ }^{\mathrm{b}}$, David K. Gardner ${ }^{\mathrm{c}}$, Alan J. Brake ${ }^{\mathrm{a}}$, Christo

G. Zouves ${ }^{a, d}$, Frank L. Barnes ${ }^{a, d}$, Manuel Viotti ${ }^{a, d, *}$

a Zouves Fertility Center, Foster City, California, USA;

${ }^{b}$ School of Biosciences, University of Kent, Canterbury, United Kingdom;

${ }^{\mathrm{c}}$ School of BioSciences, University of Melbourne, Parkville, Victoria, Australia;

d Zouves Foundation for Reproductive Medicine, Foster City, California, USA.

${ }^{*}$ Corresponding author information:

E-mail: manuel@zouvesfoundation.org

Telephone: ++16467841008

Address: Zouves Foundation for Reproductive Medicine, 1241 E Hillsdale Blvd, Suite 100, Foster City, CA 94131, USA

Manuel Viotti is Senior Scientist at the Zouves Foundation for Reproductive Medicine. Dr. Viotti heads a research laboratory working on improving outcomes in the IVF clinic, with a special focus on molecular and cell biology of the embryo and uterine environment.

\section{ABSTRACT}

Research Question: Conflicting data exists on the utility of mitochondrial DNA (mtDNA) level quantitation as a predictor of blastocyst implantation in the IVF clinic. 
Here, we determined whether blastocysts with highly elevated mitochondrial DNA (mtDNA) levels can result in healthy pregnancies and births, and whether mitochondrial functional output might be a readout of cell stress in the embryo.

Design: We determined mtDNA levels in 109 blastocysts used in clinical transfers into 100 patients, noting their clinical outcomes. In a separate set of embryos, we quantified mitochondrial function in a model of embryo stress, aneuploidy. Measurement of mtDNA levels made use of surplus material from the preimplantation genetic testing (PGT-A) process, and followed recently proposed unifying guidelines for mtDNA quantitation.

Results: Unusually high mtDNA levels did not preclude blastocyst implantation and healthy births. Analysis of 109 blastocysts showed a statistically insignificant $(P=0.231)$ difference between mtDNA levels in implanted $(n=55)$ versus nonimplanted $(n=54)$ blastocysts. We could not detect obvious differences in degree of mitochondrial functional output in a model of embryo stress.

Conclusions: Measurement of mtDNA copy number might not provide any advantage to embryo prioritization and could lead to de-selection of blastocysts that would result in healthy pregnancies and births. Furthermore, the quantitation of mitochondrial functional output in a model of cellular stress might suggest that mitochondria are not clear targets for biomarker identification as it relates to 
blastocyst viability. Any suggested link between mtDNA levels, mitochondria, or their output with blastocyst transfer outcome requires further validation.

\section{KEY MESSAGE}

Blastocysts containing highly elevated mtDNA levels can result in healthy pregnancies and births when selected for clinical transfer.

\section{KEYWORDS}

mitochondria, mtDNA, implantation, biomarker, blastocyst

\section{INTRODUCTION}

In vitro fertilization (IVF) has made it possible for many patients experiencing infertility to achieve pregnancy, but in spite of tremendous advances since its inception 40 years ago, the process remains relatively inefficient (Niederberger et al., 2018). On average, only $37.1 \%$ of embryo transfers result in implantation at IVF programs based in the USA according to the Society for Assisted Reproductive Technology (SART). Efforts to increase the likelihood of implantation and establishment of a healthy pregnancy include the identification of a biomarker predictive of viability. An ideal biomarker is a parameter that shows variability in the general embryo population that, when measured, provides a significant degree of predictive power of an embryo's chances to implant. Embryo metabolism has long been linked to viability and embryo health (Gardner and Leese, 1987; Gardner and Wale, 2013). Using 
metabolism to rank embryos within a patient's cohort could effectively increase the chances of success with fewest possible attempts (Gardner et al., 2011).

Mitochondria not only contribute significantly to the energy production for many cellular processes in the form of adenosine triphosphate (ATP), they also regulate apoptosis, calcium signalling, management of reactive oxygen species pyruvate and citric acid cycle, heme and steroid synthesis, and hormonal signalling (Harvey, 2019). Each human cell may contain a wide range in the number of mitochondrial organelles and each mitochondrion can contain numerous copies of mitochondrial DNA (mtDNA) (Lima et al., 2018). MtDNA is a circular molecule comprising $16.6 \mathrm{~kb}$ encoding 37 genes necessary for mitochondrial function. Due to its multicopy nature, mtDNA has typically been quantified on a per cell basis. That value is an assessment of 'mtDNA levels' that can be compared between any discreet populations of cells, such as individual human embryos or cellular biopsies thereof. It is nevertheless important to note that the value of mtDNA levels does not necessarily reflect the actual number of mitochondria.

Four recent studies stemming from two separate groups reported that trophectoderm (TE) biopsies from blastocysts that successfully implanted showed lower mtDNA levels on average, compared to blastocysts that did not implant after transfer (Diez-Juan et al., 2015; Fragouli et al., 2017; Fragouli et al., 2015; Ravichandran et al., 2017). Interestingly, those studies also described a threshold of mtDNA levels that, when surpassed, always resulted in implantation failure. The authors proposed that elevated mtDNA levels could be a feature of blastocysts experiencing energetic stress. It was hypothesized that stressed embryonic cells 
would promote mtDNA replication in a concerted effort to increase rates of cellular respiration and ATP production to meet increased energetic demands. Fragouli and colleagues suggested coupling this model to the the Quiet Embryo Hypothesis, which predicts that under ideal conditions an embryo experiences a calm metabolism (Leese, 2002). However, all data for the Quiet Embryo Hypothesis were collected under conditions of oxidative stress ( $20 \%$ oxygen), and consequently this hypothesis has been shown to be invalid for embryos cultured in physiological oxygen concentrations (5\%) (Gardner and Wale, 2013).

The interest generated by such reports encouraged numerous groups to explore the matter of mtDNA levels in their respective clinics. An independent study found no statistically significant correlation between mtDNA levels and implantation potential in blastocysts (Victor et al., 2017). Those findings were also confirmed in a subsequent report analysing double embryo transfers (Treff et al., 2017), which revealed that blastocysts with lower mtDNA levels were just as likely to implant than their paired counterparts with higher mtDNA levels. Subsequently, a further two published studies failed to observe any statistically significant predictive power of mtDNA levels in regard to implantation in human blastocysts (Klimczak et al., 2018; Shang et al., 2018)

Several opinions have been expressed attempting to explain the disparate results (Barnes et al., 2017; Humaidan et al., 2018; Viotti et al., 2017; Wells et al., 2017). Recently, a Views and Reviews piece focused on the possibility that technical variability in methods of mtDNA quantitation might be the reason for discrepancies, proposing guidelines to promote uniformity and reducing chance of error (Wells, 
2017). Those were: I) avoiding the use of Next Generation Sequencing platforms with low coverage of the mtDNA genome, II) using polymerase chain reaction (qPCR) targeting a mitochondrial region and a multicopy nuclear region for correct normalization to prevent allele-drop-out issues during analysis, and III) excluding samples of DNA material that may have degraded due to long-term frozen storage (Wells, 2017).

Consequently, we adhered to the proposed guidelines of mtDNA quantitation when analysing mtDNA levels in a set of blastocysts used for transfer. In doing so, it was possible to test whether technical variations in mtDNA quantitation were responsible for conflicting reports regarding the usefulness of mtDNA levels as a biomarker for implantation. The clinical outcomes of all tested blastocysts through implantation, pregnancy, and birth were followed, and further it was established whether mitochondrial function in blastocysts correlated with cellular stress.

\section{MATERIALS AND METHODS}

\section{Patients and Embryos}

Embryos derived from patients seeking infertility treatment at a private IVF center were generated by intracytoplasmic sperm injection (ICSI) and cultured to the blastocyst stage under $5 \%$ oxygen, using standard techniques, as previously described (Victor et al., 2017). Blastocysts were evaluated with the Gardner system (Gardner and Schoolcraft, 1999) and subjected to a 5-10 cell TE biopsy and vitrified until further use. Preimplantation genetic testing for aneuploidy (PGT-A) was performed in-house utilizing the VeriSeq kit (Illumina) following the manufacturer's protocol. Samples were sequenced on a MiSeq system (Illumina). Blastocysts 
classified as euploid were selected for transfer, and the presence of a gestational sac observed by endovaginal ultrasound at 3-5 weeks after transfer was considered evidence of implantation. The imaging experiments were performed on supernumerary embryos donated to research by informed consent. This study was approved by the Zouves Foundation IRB (OHRP IRB00011505, Protocol \#0003).

\section{mtDNA Quantitation}

During the PGT-A process, isolated DNA from individual TE biopsies was multiplied by whole genome amplification (WGA) and subsequently sequenced. Surplus WGA material from each sample was used to determine mtDNA levels by qPCR on a QuantStudio 3 instrument (Thermo Fisher). A 1:10 dilution of WGA product in water was vortexed for $30-60$ seconds followed by heating to $95^{\circ} \mathrm{C}$ for 10 minutes in order to inactivate any residual WGA polymerase activity. Two microliters were used in a Taqman Fast Advanced Master Mix reaction (Thermo Fisher), with each reaction run in technical triplicates. Each reaction plate contained an equilibrator sample, used for global normalization across the entire experiment.

A mitochondrial locus was quantified with a commercially available Taqman assay for the mitochondrial ND6 gene (Hs02596879_g1), which had been previously thoroughly validated in the context of blastocyst TE biopsies (Treff et al., 2017; Victor et al,, 2017). A multicopy nuclear DNA sequence was quantified using a previously described, custom-made Taqman assay targeting the Ya5 subfamily of Alu repeats (Nicklas and Buel, 2006; Treff et al., 2011), which is present in at least 2473 copies in 
the human genome. Details for the Alu-Ya5 assay were: forward primer gaccatcccggctaaaacg, reverse primer cgggttcacgccattctc, and probe cccogtctctactaaa.

ND6 and Alu-Ya5 assays yielded a cycle threshold $(C t)$ value, which was used to determine the normalized mtDNA level for any sample as follows: $2^{-(C t N D 6-C t A / u)}$. Hence, the final value for mtDNA level is a 'per cell' measure.

\section{Fluorescent Staining of Whole Blastocysts}

Supernumerary thawed blastocysts were allowed to equilibrate in an incubator for 24 $\mathrm{h}$, after which media was replaced with fresh media containing $250 \mathrm{nM}$ of MitoTracker Deep Red FM (Thermo Fisher \#M22426). After 30 min in the incubator, blastocysts were processed for immunofluorescence following a previously described protocol (Victor et al., 2018), using the primary antibodies (abs) mouse anti-human GATA3 monoclonal ab (Thermo Fisher \#MA1-028) and rabbit anti-human OCT4A monoclonal ab (Cell Signaling \#2890) followed by the secondary abs goat anti-rabbit IgG AlexaFluor488 (Thermo Fisher A11008) and goat anti-mouse IgG AlexaFluor546 (Thermo Fisher A11030). Subsequently, blastocysts were exposed to nuclear stain (Hoechst 33342, Thermo Fisher H3570) and imaged.

\section{Imaging and Computational Quantitation of Mitochondrial Function}

Stained blastocysts were placed in glass bottom dishes (MatTek P35G-1.5-20-C) in small drops of stain buffer overlaid with mineral oil (Sigma M5904), and imaged with a LSM 780 Confocal microscope (Zeiss). Image files in the .Ism format were 
uploaded into the software package Imaris 8.4.1 (Bitplane). Fluorescent channels were individually quantified in a blinded fashion computationally for each blastocyst using uniform parameters for all samples, employing background subtraction for normalization.

\section{Statistics}

Analysis and graph preparation was done in Prism 6 (GraphPad). In Fig. 2A, differences between groups were assessed by unpaired, two-tailed Student's test with Welch's correction. In Fig. 3C, differences were assessed by one-way ANOVA. For all analyses, significance was defined when $\mathrm{P}<0.05$.

\section{RESULTS}

\section{Quantifying mtDNA in Blastocysts}

To conform to the recently suggested guidelines for mtDNA quantitation in TE biopsies of human embryos (Wells, 2017), we used qPCR on TE-derived surplus WGA material stored at $-80 \mathrm{C}$ for less than 2 months without any freeze-thaw cycles. The qPCR targeted a region in the mtDNA sequence (ND6) and a multicopy region in the nuclear DNA (Alu-Ya5), using the latter as a reference to normalize for technical variability in the WGA run and number of cells in collected biopsy. To validate the assays, we performed 10-fold dilution series of a WGA sample from a TE biopsy of a euploid embryo to determine the following qPCR reaction efficiencies: $97.7 \%$ for ND6 and $97.1 \%$ for Alu-Ya5 (Fig. 1). 


\section{MtDNA Levels Have No Predictive Power Relating to Implantation}

Average levels of mtDNA were not statistically different between the set of euploid blastocysts that achieved implantation and those that did not $(P=0.231)$.

(Fig. 2A). The majority of blastocysts had mtDNA levels within a range of substantial overlap between implanted and not implanted groups. Both groups also contained outliers that could be regarded as having elevated mtDNA levels. In the implanted group, there were three blastocysts with mtDNA levels just above one standard deviation of the mean, and two blastocysts with considerably higher mtDNA levels (together, those five blastocysts are labeled \#1-5 in Figure 2A and Table 1).

\section{Blastocysts With Elevated mtDNA Levels Can Result In Healthy Pregnancies and Births}

The transfers of blastocysts \#1-5 resulted in normal pregnancies and live births. Blastocyst \#1 was particularly noteworthy for its disproportionately high mtDNA levels $(\sim 4 x$ the group average). It was also the blastocyst with highest mtDNA levels within its respective patient cohort (Figure 1B), which means that it would not have been chosen for transfer had mtDNA levels been used for de-deselection of embryos for transfer. It had good overall morphology at the time of TE biopsy (4AA) and after a vitrification-thaw cycle, immediately before transfer (5AA) (Figure 1C).

All five babies resulting from this group of blastocysts with elevated mtDNA levels passed a routine neonate physical examination and were normal for a series of 
screened conditions by blood test, including various metabolic disorders (for a list of the 63 tested conditions see Table 2).

\section{Cellular Stress Associated with Aneuploidy Does Not Necessarily Lead To Increased Mitochondrial Activity in Blastocysts}

We investigated the biological mechanism proposed by other groups explaining why elevated mtDNA levels and implantation failure might be correlated. It postulated that mtDNA levels might increase as part of a compensatory mechanism employed by embryonic cells when experiencing energetic stress (Diez-Juan et al., 2015; Fragouli et al., 2015). MtDNA levels might therefore be a corollary of a larger program that increases mitochondrial organelle number, as well as mitochondrial functional output. Accordingly, mitochondrial activity should be a more direct readout of the proposed embryo stress, and could be a potentially better biomarker of viability.

To test this possibility, we exposed blastocysts to MitoTracker Deep Red (MTDR) (Fig. 3A and B, Video 1). This fluorescent dye is associated with active mitochondria and its accumulation is dependent upon inner mitochondrial membrane potential, which increases with cellular respiration. Quantitation of MTDR fluorescent signal is therefore an indirect indicator of mitochondrial activity (Hallap et al., 2005; Kanno et al., 2016; Kodiha et al., 2015). In addition, on the same blastocysts we performed immunofluorescence with antibodies targeting a TE marker (GATA3) and an ICM marker (OCT4) to be able to differentiate mitochondrial function in the two cell lineages (Fig. 3B). 
To model the impact of cell stress, we analyzed mitochondrial function in blastocysts that had different classifications after PGT-A testing. Aneuploidy is known to be associated with global increases in cellular trauma including metabolic, proteotoxic, replicative, and mitotic cell stress (Sheltzer, 2013; Stingele et al., 2012; Zhu et al., 2018). Accordingly, euploid blastocysts were expected to exhibit the least cellular stress, followed by mosaic blastocysts, then by blastocysts with aneuploidy affecting a single chromosome, and lastly by blastocysts with aneuploidy affecting multiple chromosomes, which would have the most cellular aberrations.

On average, mitochondrial activity per cell in the TE was considerably higher than in the ICM, in agreement with previously reported data (Van Blerkom, 2011). Nonetheless, we noted no statistically significant difference regarding mitochondrial activity per cell when comparing the different blastocysts groups $(P=0.958$ in the TE, $\mathrm{P}=0.946$ in the ICM) (Figure 3C).

\section{DISCUSSION}

Biomarkers predictive of implantation are urgently needed to reduce time to pregnancy and to help establish healthy pregnancies in IVF (Ferrick et al., 2019; Gardner et al., 2015). Here we tested whether mtDNA levels possessed prognostic qualities regarding blastocyst transfer outcome in our clinic, abiding to suggested guidelines of mtDNA quantitation (Wells, 2017). We found no association between mtDNA levels and blastocyst viability, and we report for the first time that blastocysts with extremely high mtDNA copy number are consistent with viable implantation, normal pregnancy, and birth. Using a unified system of mtDNA quantitation, we exclude the possibility that technical differences were responsible for reported 
discrepancies between our findings and those previously describing a prognostic value of mtDNA levels in blastocysts (Diez-Juan et al., 2015; Fragouli et al., 2017; Fragouli et al., 2015; Ravichandran et al., 2017; Victor et al., 2017).

One scenario that reconciles the conflicting reports is a laboratory-selective association between mtDNA levels and implantation. In fact, it has been reported that the incidence of blastocyst with elevated mtDNA levels varies widely amongst IVF centers (Ravichandran et al., 2017). Out of 35 clinics participating in that study, roughly half did not produce an appreciable percentage of blastocysts with high mtDNA levels, although it must be noted that for some centers the analyzed sample size was small. In the remaining centers, incidences of blastocysts with elevated mtDNA levels ranged from $1 \%$ to $27 \%$. Of blastocysts analyzed in this study, three out of $109(2.8 \%)$ could be considered as containing highly elevated mtDNA levels (two implanted, one did not), and yet mtDNA quantitation was not valuable in predicting implantation in our setting.

One can only speculate why those three embryos contained highly elevated mtDNA levels. Under normal circumstances, no new mtDNA replication occurs between zygote and blastocyst stage, such that the initial set mtDNA molecules becomes split between dividing cells and progressively diluted amongst cells of the developing embryo (Cecchino et al., 2018; St John et al., 2010). Therefore, if a cell fails to undergo one or more cell divisions, it will tend to retain its mtDNA content. It is possible that for the three blastocysts yielding highly elevated mtDNA levels the collected biopsy happened to contain a region of the TE that had undergone fewer cell divisions, resulting in concentrated mtDNA content compared to other TE 
regions. The blastocyst with the highest mtDNA levels, which contained fourfold the content compared to the group average, could be explained if the sampled TE cells had skipped two cell divisions compared to other TE regions of that blastocyst. What might have caused attenuated cell division in that part of the TE is another question, although to the best of our knowledge there is no clear evidence suggesting that all TE regions proliferate at equal rates under normal conditions. For example, TE cells adjacent to the section of the zona pellucida making contact with the dish during culture might experience distinct cell proliferation dynamics.

Alternatively, it has been shown in several mammalian species that once the blastocyst stage is reached, replication of mtDNA begins specifically in the TE while the ICM continues to reduce its mtDNA copy number (St John et al., 2010; Van Blerkom, 2011). Whether mtDNA replication occurs uniformly across TE cells or in a localized fashion is not well studied, and might be affected by the cell cycle stage of each individual TE cell. A spurt of mtDNA replication in a sampled region would result in high mtDNA levels being quantified for the entire corresponding embryo.

MtDNA levels have been proposed to be a surrogate measure of mitochondrial function, which was presumed to increase in blastocysts experiencing stress (DiezJuan et al., 2015; Fragouli et al., 2015). Nonetheless, a direct relationship between mtDNA levels and mitochondrial function in the blastocysts has not been documented (Van Blerkom, 2004). To shed light on this matter, mitochondrial function in blastocysts euploid and aneuploid blastocysts were analysed. Even though the data suggest that ploidy does not affect mitochondrial function in blastocysts, it is possible that anueploidy is not representative of the type of stress that might elicit 
mitochondrial activation in blastocysts, such as for example changes in oxygen concentration (Gardner and Harvey, 2015). The state of aneuploidy (the model tested here) can elicit increases in various types of cell stress (metabolic, proteotoxic, replication, and mitotic) (Zhu et al., 2018), but it is possible that only a specific subtype of energetic stress prompts mitochondrial activation, and consequently shifts in mtDNA levels. What is more, the trigger might depend on a specific threshold of that stress factor. Future studies need to test whether lab-induced variance of parameters including oxygen concentration, medium composition, $\mathrm{pH}$, and/or temperature affect mitochondrial function and/or content in human embryos. This in turn could help to explain the observation that different centers generate vastly different incidences of blastocysts with highly elevated mtDNA levels (Ravichandran et al., 2017), a phenomenon for which the underlying biological basis remains unknown. We surmise that for the time being, the suggested rationale linking increased mtDNA levels to stress remains purely speculative.

\section{CONCLUSIONS}

Based upon the results of this study that conforms to proposed guidelines of mtDNA quantitation, mtDNA levels were not predictive of implantation potential. Importantly, blastocysts with disproportionally elevated mtDNA levels resulted in healthy pregnancies and births. The functional output of mitochondria is not significantly elevated in a model of embryo stress, refuting the previously suggested model that links stress and mtDNA. We conclude that mtDNA copy number is not a universal 
biomarker of implantation. Future studies are needed to investigate in which cases mtDNA quantitation may be useful.

\section{Acknowledgements}

We are grateful to the entire staff of Zouves Fertility Center for their contributions to this work.

\section{Funding}

The study was funded by the Zouves Foundation for Reproductive Medicine and Zouves Fertility Center. 


\section{VIDEO LEGENDS}

Supplemental Video 1. Video of confocal microscopy-imaged embryo after immunofluorescence staining, displaying quantitation method. This representative sample was classified as euploid with PGT-A. Note that the image analysis software detects the concrete number (count) of nuclei (blue), ICM cells (white), TE cells (red). MTDR signal (green) is quantified as fluorescent signal intensity (arbitrary units - AU) within a 'mask' generated by the software. 


\section{BIBLIOGRAPHY}

Barnes, F. L., Victor, A. R., Zouves, C. G., and Viotti, M. (2017). Mitochondrial DNA quantitation-making sense of contradictory reports. Hum Reprod 32, 2149-2150.

Cecchino, G. N., Seli, E., Alves da Motta, E. L., and Garcia-Velasco, J. A. (2018). The role of mitochondrial activity in female fertility and assisted reproductive technologies: overview and current insights. Reprod Biomed Online 36, 686-697.

Diez-Juan, A., Rubio, C., Marin, C., Martinez, S., Al-Asmar, N., Riboldi, M., DiazGimeno, P., Valbuena, D., and Simon, C. (2015). Mitochondrial DNA content as a viability score in human euploid embryos: less is better. Fertil Steril 104, 534-541 e531.

Ferrick, L., Lee, Y. S. L., and Gardner, D. K. (2019). Reducing time to pregnancy and facilitating the birth of healthy children through functional analysis of embryo physiology. Biol Reprod.

Fragouli, E., McCaffrey, C., Ravichandran, K., Spath, K., Grifo, J. A., Munne, S., and Wells, D. (2017). Clinical implications of mitochondrial DNA quantification on pregnancy outcomes: a blinded prospective non-selection study. Hum Reprod 32, 2340-2347.

Fragouli, E., Spath, K., Alfarawati, S., Kaper, F., Craig, A., Michel, C. E., Kokocinski, F., Cohen, J., Munne, S., and Wells, D. (2015). Altered levels of mitochondrial DNA are associated with female age, aneuploidy, and provide an independent measure of embryonic implantation potential. PLoS Genet 11, e1005241.

Gardner, D. K., and Harvey, A. J. (2015). Blastocyst metabolism. Reprod Fertil Dev 27, 638-654.

Gardner, D. K., and Leese, H. J. (1987). Assessment of embryo viability prior to transfer by the noninvasive measurement of glucose uptake. J Exp Zool 242, 103105.

Gardner, D. K., Meseguer, M., Rubio, C., and Treff, N. R. (2015). Diagnosis of human preimplantation embryo viability. Hum Reprod Update 21, 727-747.

Gardner, D. K., and Schoolcraft, W. B. (1999). In vitro culture of human blastocysts. . In Towards reproductive certainty: fertility and genetics beyond, J. R., and M. D., eds. (Camforth, UK: Parthenon Publishing), pp. 378-388. 
Gardner, D. K., and Wale, P. L. (2013). Analysis of metabolism to select viable human embryos for transfer. Fertil Steril 99, 1062-1072.

Gardner, D. K., Wale, P. L., Collins, R., and Lane, M. (2011). Glucose consumption of single post-compaction human embryos is predictive of embryo sex and live birth outcome. Hum Reprod 26, 1981-1986.

Hallap, T., Nagy, S., Jaakma, U., Johannisson, A., and Rodriguez-Martinez, H. (2005). Mitochondrial activity of frozen-thawed spermatozoa assessed by MitoTracker Deep Red 633. Theriogenology 63, 2311-2322.

Harvey, A. J. (2019). Mitochondria in early development: linking the microenvironment, metabolism and the epigenome. Reproduction 157, R159-R179.

Humaidan, P., Kristensen, S. G., and Coetzee, K. (2018). Mitochondrial DNA, a new biomarker of embryonic implantation potential: fact or fiction? Fertil Steril 109, 61-62.

Kanno, C., Kang, S. S., Kitade, Y., Yanagawa, Y., Takahashi, Y., and Nagano, M. (2016). Simultaneous evaluation of plasma membrane integrity, acrosomal integrity, and mitochondrial membrane potential in bovine spermatozoa by flow cytometry. Zygote 24, 529-536.

Klimczak, A. M., Pacheco, L. E., Lewis, K. E., Massahi, N., Richards, J. P., Kearns, W. G., Saad, A. F., and Crochet, J. R. (2018). Embryonal mitochondrial DNA: relationship to embryo quality and transfer outcomes. J Assist Reprod Genet 35, 871877.

Kodiha, M., Pie, B., Wang, Y. M., Flamant, E., Boppana, N. B., Young, J. C., Separovic, D., Cooper, E., and Stochaj, U. (2015). Detecting changes in the mitochondrial membrane potential by quantitative fluorescence microscopy. Protocol Exchange.

Leese, H. J. (2002). Quiet please, do not disturb: a hypothesis of embryo metabolism and viability. Bioessays 24, 845-849.

Lima, A., Burgstaller, J., Sanchez-Nieto, J. M., and Rodriguez, T. A. (2018). The Mitochondria and the Regulation of Cell Fitness During Early Mammalian Development. Curr Top Dev Biol 128, 339-363.

Nicklas, J. A., and Buel, E. (2006). Simultaneous determination of total human and male DNA using a duplex real-time PCR assay. J Forensic Sci 51, 1005-1015.

Niederberger, C., Pellicer, A., Cohen, J., Gardner, D. K., Palermo, G. D., O'Neill, C. L., Chow, S., Rosenwaks, Z., Cobo, A., Swain, J. E., et al. (2018). Forty years of IVF. Fertil Steril 110, 185-324 e185.

Ravichandran, K., McCaffrey, C., Grifo, J., Morales, A., Perloe, M., Munne, S., Wells, D., and Fragouli, E. (2017). Mitochondrial DNA quantification as a tool for embryo 
viability assessment: retrospective analysis of data from single euploid blastocyst transfers. Hum Reprod 32, 1282-1292.

SART Society for Reproductive Technology, National Summary Report. https://wwwsartcorsonlinecom/rptCSR PublicMultYearaspx?reportingYear=2016.

Shang, W., Zhang, Y., Shu, M., Wang, W., Ren, L., Chen, F., Shao, L., Lu, S., Bo, S., Ma, S., and Gao, Y. (2018). Comprehensive chromosomal and mitochondrial copy number profiling in human IVF embryos. Reprod Biomed Online 36, 67-74.

Sheltzer, J. M. (2013). A transcriptional and metabolic signature of primary aneuploidy is present in chromosomally unstable cancer cells and informs clinical prognosis. Cancer Res 73, 6401-6412.

St John, J. C., Facucho-Oliveira, J., Jiang, Y., Kelly, R., and Salah, R. (2010). Mitochondrial DNA transmission, replication and inheritance: a journey from the gamete through the embryo and into offspring and embryonic stem cells. Hum Reprod Update 16, 488-509.

Stingele, S., Stoehr, G., Peplowska, K., Cox, J., Mann, M., and Storchova, Z. (2012). Global analysis of genome, transcriptome and proteome reveals the response to aneuploidy in human cells. Mol Syst Biol 8, 608.

Treff, N. R., Su, J., Taylor, D., and Scott, R. T., Jr. (2011). Telomere DNA deficiency is associated with development of human embryonic aneuploidy. PLoS Genet 7, e1002161.

Treff, N. R., Zhan, Y., Tao, X., Olcha, M., Han, M., Rajchel, J., Morrison, L., Morin, S. J., and Scott, R. T., Jr. (2017). Levels of trophectoderm mitochondrial DNA do not predict the reproductive potential of sibling embryos. Hum Reprod 32, 954-962.

Van Blerkom, J. (2004). Mitochondria in human oogenesis and preimplantation embryogenesis: engines of metabolism, ionic regulation and developmental competence. Reproduction 128, 269-280.

Van Blerkom, J. (2011). Mitochondrial function in the human oocyte and embryo and their role in developmental competence. Mitochondrion 11, 797-813.

Victor, A. R., Brake, A. J., Tyndall, J. C., Griffin, D. K., Zouves, C. G., Barnes, F. L., and Viotti, M. (2017). Accurate quantitation of mitochondrial DNA reveals uniform levels in human blastocysts irrespective of ploidy, age, or implantation potential. Fertil Steril 107, 34-42 e33.

Victor, A. R., Griffin, D. K., Brake, A. J., Tyndall, J. C., Murphy, A. E., Lepkowsky, L. T., Lal, A., Zouves, C. G., Barnes, F. L., McCoy, R. C., and Viotti, M. (2018).

Assessment of aneuploidy concordance between clinical trophectoderm biopsy and blastocyst. Hum Reprod. 
Viotti, M., Victor, A. R., Zouves, C. G., and Barnes, F. L. (2017). Is mitochondrial DNA quantitation in blastocyst trophectoderm cells predictive of developmental competence and outcome in clinical IVF? J Assist Reprod Genet 34, 1581-1585.

Wells, D. (2017). Mitochondrial DNA quantity as a biomarker for blastocyst implantation potential. Fertil Steril 108, 742-747.

Wells, D., Ravichandran, K., McCaffrey, C., Grifo, J., Morales, A., Perloe, M., Munne, S., and Fragouli, E. (2017). Reply: Mitochondrial DNA Quantification-the devil in the detail. Hum Reprod 32, 2150-2151.

Zhu, J., Tsai, H. J., Gordon, M. R., and Li, R. (2018). Cellular Stress Associated with Aneuploidy. Dev Cell 44, 420-431. 


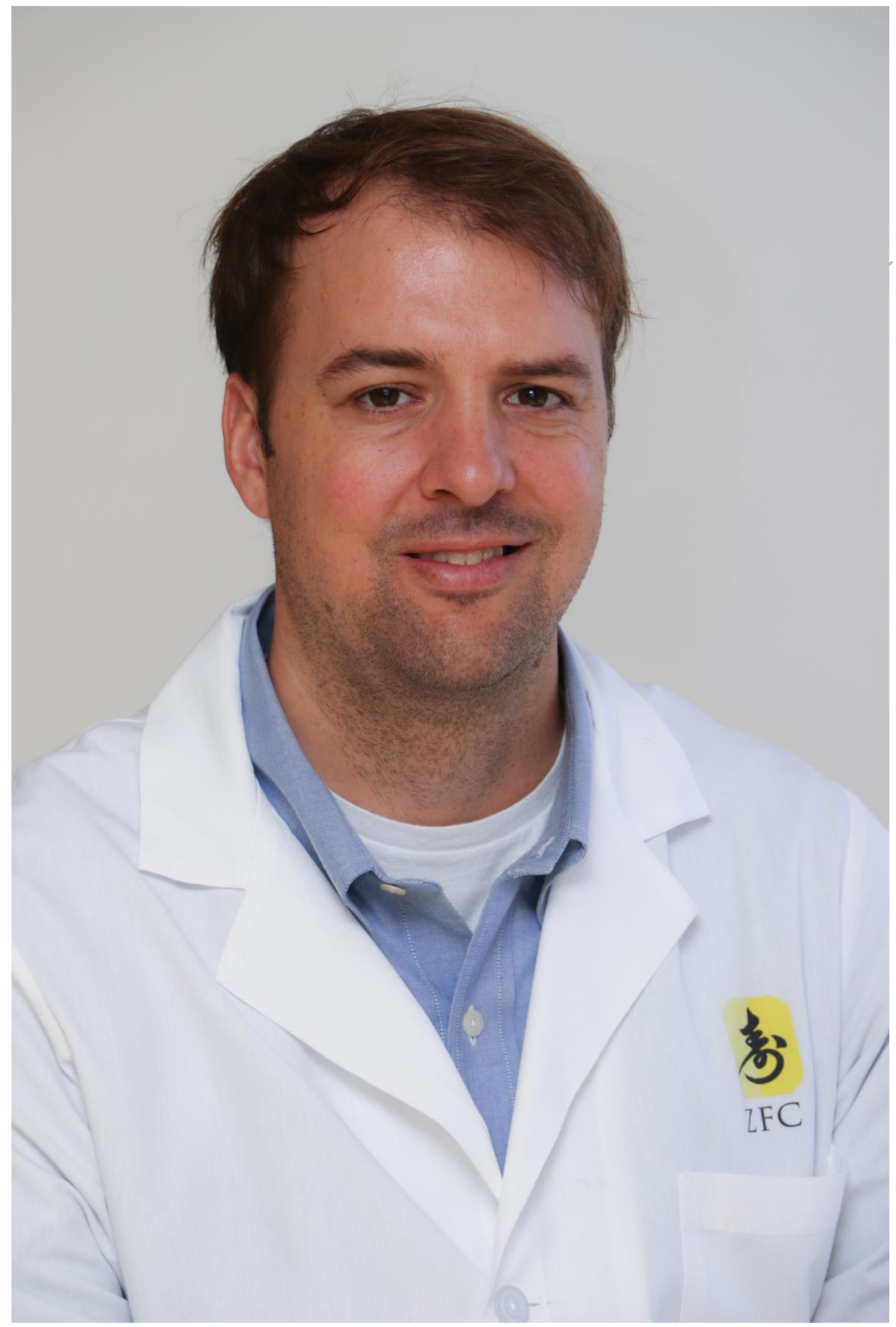




\section{Figure 1.}

Technical validation of qPCR assays used in this study to quantify mtDNA levels per cell.

Graphs depict regression curves of serial dilution experiments.
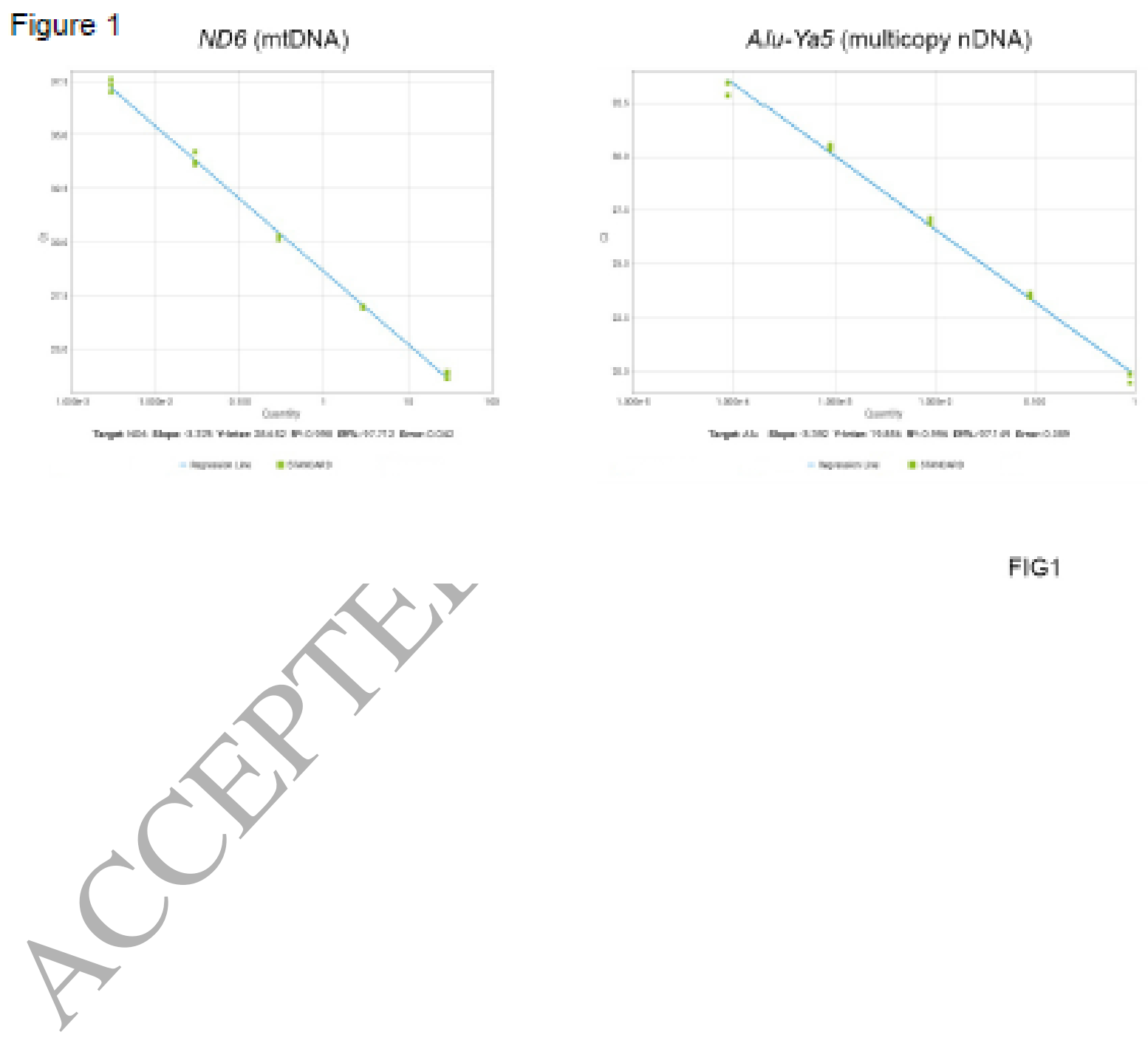

FIG1 


\section{Figure 2.}

MtDNA levels are not predictive of implantation in the analyzed cohort.

(A) Comparison of mtDNA levels between blastocysts that implanted and those that did not. Each data point represents the mtDNA levels of one blastocyst.

(B) Plotted mtDNA levels of all euploid blastocysts from patient who generated blastocyst \#1 in $(A)$.

(C) Images of blastocyst \#1 in (A), immediately before collection of a TE biopsy and vitrification (left image), and before transfer (right image). 


\section{Fonor}

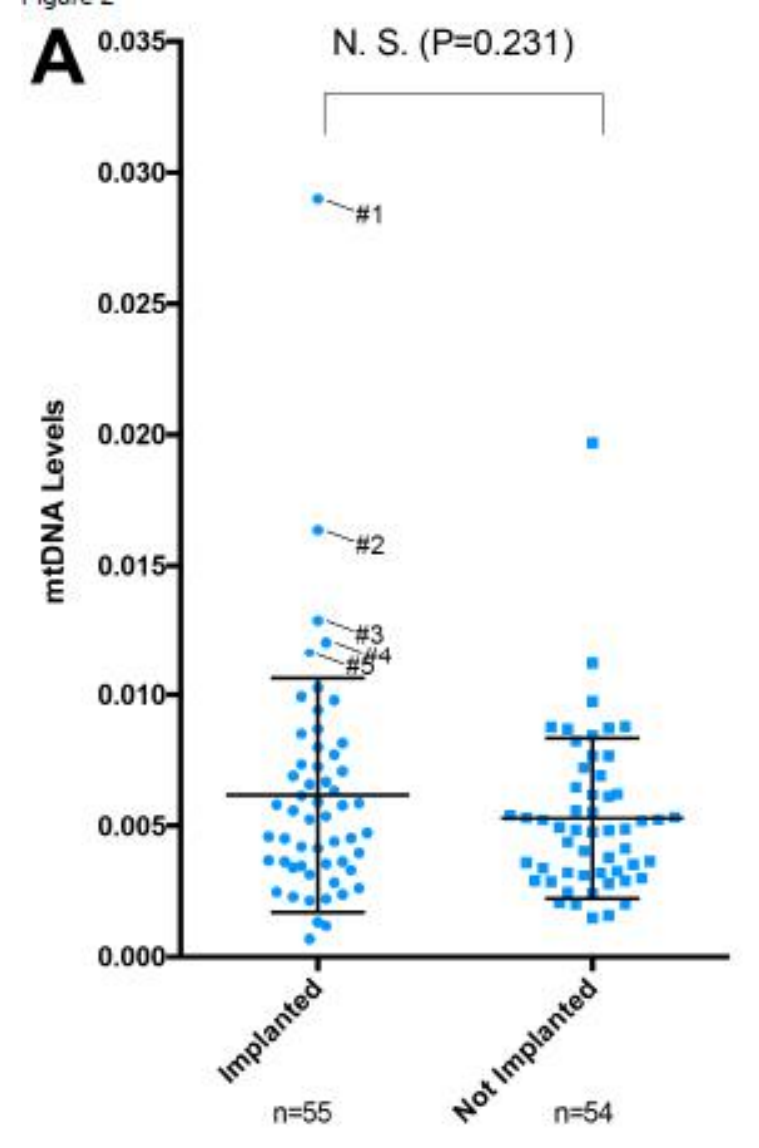

C
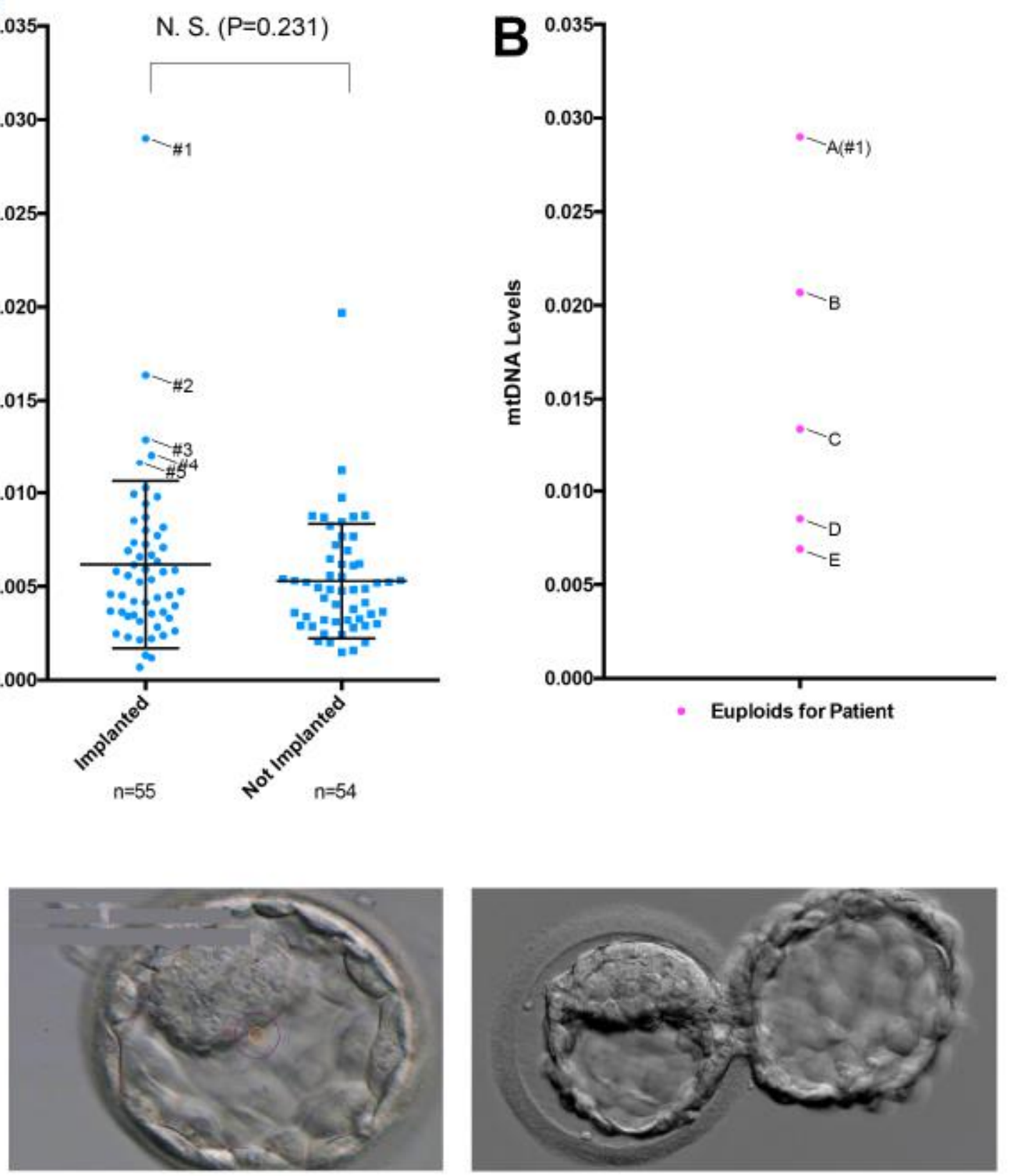


\section{Figure 3.}

\section{Quantitation of mitochondrial activity in blastocysts.}

(A) High resolution image of TE cells after exposure to Hoechst nuclear stain and MTDR, displaying fluorescent MTDR signal in characteristic punctate distribution around nuclei. Scale bar $=10 \mu \mathrm{m}$

(B) Left column shows representative immunofluorescent images of a whole hatching blastocyst. Right column shows method of computational detection and quantitation Note that the image analysis software detects the concrete number (count) of nuclei, ICM cells, and TE cells. The software generates a 'mask' of the MTDR signal, and quantifies the fluorescence within. The yellow number at the bottom right corner of panels indicates the computed value for the corresponding image. Scale bar $=30 \mu \mathrm{m}$

(C) Scatter dot plots depicting quantitation of MTDR fluorescence (readout of mitochondrial function) in blastocysts. Each symbol represents one blastocyst. Lines indicate mean with standard deviation. Sample size of each blastocyst group is $n=4$ Euploids, $\mathrm{n}=4$ Mosaics, $\mathrm{n}=8$ Single Aneuploids (aneuploidy affecting a single chromosome), $\mathrm{n}=7$ Multiple Aneuploids (with aneuploidy affecting multiple chromosomes). ${ }^{*}, \mathrm{P}<0.05$; N.S. (not significant), $\mathrm{P} \geq 0.05$. 


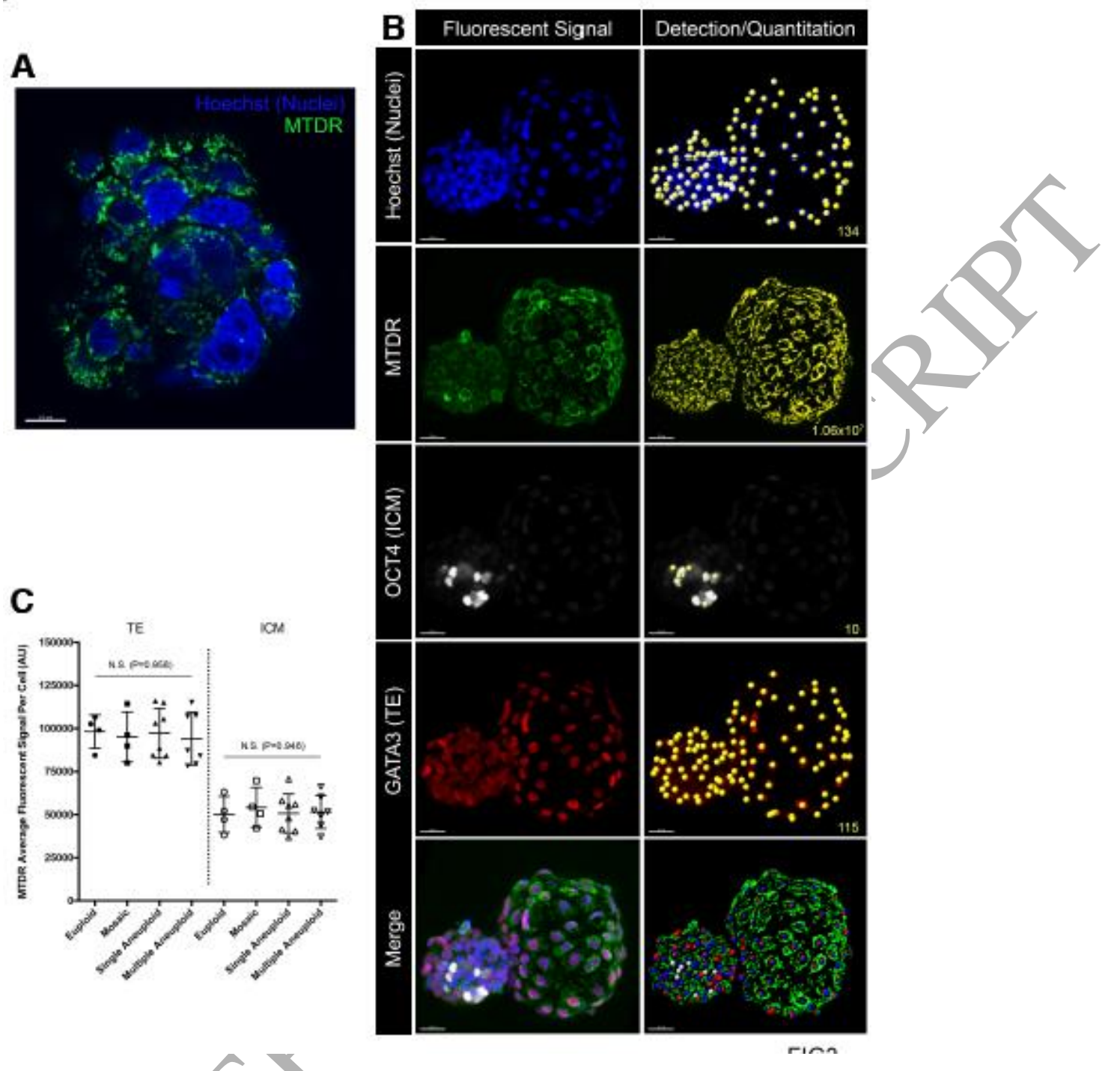


Table 1. List of implanted euploid blastocysts with highest mtDNA levels.

\begin{tabular}{|c|c|c|c|c|c|c|c|}
\hline $\begin{array}{c}\text { Embryo } \\
\text { Number }\end{array}$ & $\begin{array}{c}\text { Gardner } \\
\text { System }\end{array}$ & $\begin{array}{c}\text { Biopsy } \\
\text { Day }\end{array}$ & $\begin{array}{c}\text { PGT-A } \\
\text { Result }\end{array}$ & $\begin{array}{c}\text { mtDNA } \\
\text { Level }\end{array}$ & $\begin{array}{c}\text { mtDNA Level } \\
\text { Compared to } \\
\text { Group Mean }\end{array}$ & Implantation & Birth \\
\hline$\# 1$ & 4AA & D5 & Normal & 0.02899 & $4.70 x$ & Yes & Yes \\
\hline$\# 2$ & 5BB & D6 & Normal & 0.01635 & $2.65 x$ & Yes & Yes \\
\hline$\# 3$ & 5BB & D6 & Normal & 0.01291 & $2.09 x$ & Yes & Yes \\
\hline$\# 4$ & 3AB & D5 & Normal & 0.01208 & $1.96 x$ & Yes & Yes \\
\hline$\# 5$ & 5BC & D6 & Normal & 0.01164 & $1.89 x$ & Yes & Yes \\
\hline
\end{tabular}


Table 2. Conditions tested in newborns resulting from transfers of blastocysts with elevated mtDNA levels.

\begin{tabular}{|c|c|}
\hline Category & Condition \\
\hline \multirow{18}{*}{$\begin{array}{l}\text { Amino Acid } \\
\text { Disorders }\end{array}$} & Argininemia (ARG) \\
\hline & Argininosuccinic Aciduria (ASA) \\
\hline & Benign Hyperphenylalaninemia (H-PHE) \\
\hline & Biopterin Defect in Cofactor Biosynthesis (BIOPT-BS) \\
\hline & Biopterin Defect in Cofactor Regeneration (BIOPT-REG) \\
\hline & Carbamoyl Phosphate Synthetase I Deficiency (CPS) \\
\hline & Citrullinemia, Type I (CIT) \\
\hline & Citrullinemia, Type II (CIT II) \\
\hline & Classic Phenylketonuria (PKU) \\
\hline & Homocystinuria $(\mathrm{HCY})$ \\
\hline & Hypermethioninemia (MET) \\
\hline & Hyperornithine with Gyrate Deficiency (Hyper ORN) \\
\hline & Maple Syrup Urine Disease (MSUD) \\
\hline & Ornithine Transcarbamylase Deficiency (OTC) \\
\hline & Prolinemia (PRO) \\
\hline & Tyrosinemia, Type I (TYR I) \\
\hline & Tyrosinemia, Type II (TYR II) \\
\hline & Tyrosinemia, Type III (TYR III) \\
\hline \multirow{2}{*}{$\begin{array}{l}\text { Endocrine } \\
\text { Disorders }\end{array}$} & Congenital Adrenal Hyperplasia (CAH) \\
\hline & Primary Congenital Hypothyroidism $(\mathrm{CH})$ \\
\hline \multirow{11}{*}{$\begin{array}{l}\text { Fatty Acid } \\
\text { Oxidation Disorders }\end{array}$} & Carnitine Acylcarnitine Translocase Deficiency (CACT) \\
\hline & Carnitine Palmitoyltransferase I Defíciency (CPT-IA) \\
\hline & Carnitine Palmitoyltransferase Type II Deficiency (CPT-II) \\
\hline & Carnitine Uptake Defect (CUD) \\
\hline & Glutaric Acidemia, Type II (GA-2) \\
\hline & Long-Chain L-3 Hydroxyacyl-CoA Dehydrogenase Deficiency (LCHAD) \\
\hline & Medium-Chain Acyl-CoA Dehydrogenase Deficiency (MCAD) \\
\hline & Medium/Short-Chain L-3 Hydroxyacyl-CoA Dehydrogenase Deficiency (M/SCHAD) \\
\hline & Short-Chain Acyl-CoA Dehydrogenase Deficiency (SCAD) \\
\hline & Trifunctional Protein Deficiency (TFP) \\
\hline & Very Long-Chain Acyl-CoA Dehydrogenase Deficiency (VLCAD) \\
\hline \multirow{4}{*}{$\begin{array}{l}\text { Hemoglobin } \\
\text { Disorders }\end{array}$} & Hemoglobinopathies (Var $\mathrm{Hb})$ \\
\hline & S, Beta-Thalassemia (Hb S/BTh) \\
\hline & C Disease $(\mathrm{Hb} \mathrm{S} / \mathrm{C})$ \\
\hline & Sickle Cell Anemia (Hb SS) \\
\hline \multirow{2}{*}{$\begin{array}{l}\text { Lysosomal Storage } \\
\text { Disorders }\end{array}$} & Mucopolysaccharidosis Type-I (MPS I) \\
\hline & Pompe (POMPE) \\
\hline \multirow{10}{*}{$\begin{array}{l}\text { Organic Acid } \\
\text { Conditions }\end{array}$} & 2-Methyl-3-Hydroxybutyric Acidemia (2M3HBA) \\
\hline & 2-Methylbutyrylglycinuria (2MBG) \\
\hline & $\begin{array}{l}\text { 3-Hydroxy-3-Methylglutaric Aciduria (HMG) } \\
\text { 3-Methylcrotonyl-CoA Carboxylase Deficiency (3-MCC) }\end{array}$ \\
\hline & 3-Methylglutaconic Aciduria (3MGA) \\
\hline & Beta-Ketothiolase Deficiency (BKT) \\
\hline & Ethylmalonic Encephalopathy (EME) \\
\hline & Glutaric Acidemia, Type I (GA-1) \\
\hline & Holocarboxylase Synthetase Deficiency (MCD) \\
\hline & Isobutyrylglycinuria (IBG) \\
\hline & Isovaleric Acidemia (IVA) \\
\hline
\end{tabular}




\begin{tabular}{|l|l|}
\hline \multirow{4}{*}{ Other Disorders } & Malonic Acidemia (MAL) \\
\cline { 2 - 2 } & Methylmalonic Acidemia (Cobalamin Disorders) (CbI A,B) \\
\cline { 2 - 2 } & Methylmalonic Acidemia (Methymalonyl-CoA Mutase Deficiency) (MUT) \\
\cline { 2 - 2 } & Methylmalonic Acidemia with Homocystinuria (CbI C, D, F) \\
\cline { 2 - 2 } & Propionic Acidemia (PROP) \\
\cline { 2 - 2 } & Adrenoleukodystrophy (ALD) \\
\cline { 2 - 2 } & Biotinidase Deficiency (BIOT) \\
\cline { 2 - 2 } & Classic Galactosemia (GALT) \\
\cline { 2 - 2 } & Critical Congenital Heart Disease (CCHD) \\
\cline { 2 - 2 } & Fystic Fibrosis (CF) \\
\cline { 2 - 2 } & Formiminoglutamic Acidemia (FIGLU) \\
\cline { 2 - 2 } & Hearing loss (HEAR) \\
\cline { 2 - 3 } & Hyperornithinemia-Hyperammonemia-Homocitrullinuria Syndrome (HHH) \\
\cline { 2 - 3 } & Severe Combined Immunodeficiency (SCID) \\
\cline { 2 - 2 } & T-cell Related Lymphocyte Deficiencies \\
\hline
\end{tabular}

